\title{
ILCEA
}

Revue de l'Institut des langues et cultures

d'Europe, Amérique, Afrique, Asie et Australie

$20 \mid 2014$

Le conte : d'un art à l'autre

\section{Des bestsellers grâce aux citations de contes? Les romans à succès de Berthold Auerbach et d'Eugenie Marlitt et leurs pré-textes issus de la poésie populaire}

Bestsellers Thanks to Fairy-tales Quotations? Berthold Auerbach's and Eugenie

Marlitt's Successfull Novels and their Pre-texts of Folk Poetry

Bestseller dank Märchenzitaten? Berthold Auerbachs und Eugenie Marlitts

Bucherfolge und ihre volkspoetischen Prätexte

Jesko Reiling

\section{OpenEdition}

Journals

Édition électronique

URL : http://journals.openedition.org/ilcea/2638

DOI : 10.4000/ilcea.2638

ISSN : 2101-0609

Éditeur

UGA Éditions/Université Grenoble Alpes

Édition imprimée

ISBN : 978-2-84310-290-5

ISSN : 1639-6073

\section{Référence électronique}

Jesko Reiling, « Des bestsellers grâce aux citations de contes ? Les romans à succès de Berthold

Auerbach et d'Eugenie Marlitt et leurs pré-textes issus de la poésie populaire », ILCEA [Online],

20 | 2014, Online erschienen am: 08 Dezember 2014, abgerufen am 21 April 2019. URL : http://

journals.openedition.org/ilcea/2638; DOI : 10.4000/ilcea.2638

Ce document a été généré automatiquement le 21 avril 2019

(C) ILCEA 


\section{Des bestsellers grâce aux citations de contes? Les romans à succès de Berthold Auerbach et d'Eugenie Marlitt et leurs pré-textes issus de la poésie populaire}

Bestsellers Thanks to Fairy-tales Quotations? Berthold Auerbach's and Eugenie

Marlitt's Successfull Novels and their Pre-texts of Folk Poetry

Bestseller dank Märchenzitaten? Berthold Auerbachs und Eugenie Marlitts

Bucherfolge und ihre volkspoetischen Prätexte

Jesko Reiling

Volksmärchen, Sagen, Legenden, Volkslieder, mittelalterliche Epen und Volksbücher waren die großen literarischen Entdeckungen des 19. Jahrhunderts. Angeregt von den vermeintlich aus keltischer Vorzeit stammenden Fragments of Ancient Poetry, den Gesängen Ossians, die James Macpherson 1760 herausgab, sowie weiteren Sammlungen von wahrhaftig oder nur scheinbar alten Dichtungen begann sich in der Epoche des Sturm und Drang ein Interesse an der Volkspoesie auszubreiten, das schon bald Publikationen hervorbrachte, die bis heute sehr geschätzt werden. Neben Herders Volksliedern von 1778-1779 und der Wunderhorn-Sammlung (1806-1808) von Achim von Arnim und Clemens Brentano ist hierbei natürlich vor allem an die Märchen- und Sagensammlungen der Brüder Grimm zu erinnern. Den Kinder- und Hausmärchen (1812) der Grimms folgte im weiteren Verlauf des 19. Jahrhunderts eine Vielzahl weiterer Märchen- oder Sagensammlungen, etwa von Ludwig Bechstein (Deutsches Märchenbuch, EA 1845), Heinrich Pröhle (Kinder- und Volksmärchen, 1853) oder Otto Sutermeister (Kinderund Hausmärchen aus der Schweiz, 1869), ebenso erfreuten sich Volkslieder-Anthologien großer Beliebtheit (etwa Ludwig Uhland: Alte hoch- und niederdeutsche Volkslieder. Liedersammlung in 5 Büchern, 2 Bde., 1844-1845; Hoffmann von Fallersleben: Unsere 
volksthümlichen Lieder, EA 1857; Ludwig Erk, Franz Magnus Böhme: Deutscher Liederhort, 3 Bde., 1893-1894). Märchen und die weiteren Formen der Natur- respektive Volkspoesie sahen die Zeitgenossen im 19. Jahrhundert als Zeugnisse einer ehrwürdigen nationalen Kultur an, aus deren Geist sie sich eine Belebung und Stärkung der eigenen Identität und gemeinschaftlichen Verbundenheit erhofften. Wohl vor allem deshalb erfuhr die Volkspoesie im 19. Jahrhundert ein solch großes literarhistorisches, kulturelles sowie dichterisches Interesse. Neben der Tradition der Volksmärchen entwickelte sich auch diejenige der Kunstmärchen, die inspiriert von ausländischen Vorbildern in Deutschland etwa die Volksmährchen der Deutschen von Johann Karl August Musäus (1782-1786), Wilhelm Hauffs Märchen-Almanache (1825-1827) oder die aus dem Dänischen übersetzten Märchen von Hans Christian Andersen hervorbrachte. Und so darf man mit Recht behaupten, dass Märchen $\mathrm{zu}$ den erfolgreichsten literarischen Produkten des 19. Jahrhunderts gehören.

2 Berthold Auerbachs Dorfgeschichte Barfüßele aus dem Jahre 1856 und Eugenie Marlitts Erzählung Goldelse von 1866 gehören ebenfalls $\mathrm{zu}$ den populärsten Werken des 19. Jahrhunderts. Auerbachs Barfüßele ist bis heute in über 120 Ausgaben und in gut 30 verschiedensprachigen Übersetzungen erschienen, wobei jeweils gut die Hälfte dieser Auflagen bereits im 19. Jahrhundert auf den Markt kam (Regenbogen, 2006). Marlitts Erfolg war ähnlich groß, wenn auch genauere bibliographische Untersuchungen hierzu bislang noch ausstehen: 1887 erschien die Goldelse als Einzeldruck bereits in 20. Auflage; zusammen mit den Nachfolgeromanen, die Marlitt stets zunächst als Fortsetzungen in der Gartenlaube, der bis heute bekanntesten Familienzeitschrift des 19. Jahrhunderts, publizierte, soll der Roman, so die gängige, aber letztlich wohl doch zu eindimensionale Erzählung, kausal die immense Auflagensteigerung der Gartenlaube bewirkt haben: Während die Gartenlaube 1860 in einer Auflage von 86000 Exemplaren erschien, so waren es 1871 rund dreieinhalb mal so viele, nämlich 310000 Exemplare (Bonter, 2005: 11).

3 Barfüßele und Goldelse sind aber nicht nur hinsichtlich ihres Popularitätsgrades vergleichbar, sie weisen auch verblüffende inhaltliche Parallelen auf: In beiden Werken ist die Hauptfigur ein junges Naturkind, ein unschuldiges Mädchen aus dem „Volk“, und in beiden Texten lässt sich eine Vielzahl an intertextuellen Bezügen zu Märchen feststellen, die aufgrund ihres gehäuften Auftretens erklärungsbedürftig sind. Es drängt sich die Frage auf: Wurden die beiden Erzählungen Bestseller, weil sie Märchenzitate enthalten? - Darauf lässt sich sinnvoll nur in spekulativer Weise antworten, und so scheint es angebracht, die Frage anders $\mathrm{zu}$ operationalisieren. Es soll deshalb im Folgenden darum gehen, Bedeutung und Funktion der Märchenzitate für beide Erzählungen $\mathrm{zu}$ bestimmen und nach Gemeinsamkeiten sowie Differenzen bei der Verwendung der intertextuellen Referenzen zu suchen. Zu untersuchen ist auch, ob sich die Rezeption der Märchen lediglich auf motivische Übernahme beschränkt oder ob sich auch poetologische Einflüsse feststellen lassen, die das Poesieverständnis der beiden Autoren auf einer abstrakt-theoretischen Ebene berühren.

4 Auerbach hatte ursprünglich die Absicht, seiner Schwarzwälder Dorfgeschichte Barfüßele, die den sozialen Aufstieg des Waisenkinds Amrei von einer einsamen Gänsemagd zur glücklich verheirateten Großbäuerin erzählt, den Titel „Das neue Aschenputtel“ zu geben (Auerbach nach Bettelheim, 1907: 254; Reiling, 2012: 97-120). Intention seiner Erzählung, so Auerbach selbst, sei es, „eine Art Aschenbrödel in realistischer Motivierung“ zu zeigen (Auerbach nach Scheuffelen, 1986: 68). Er entschied sich dann aber doch für den Titel Barfüßele, und dies aus gutem Grund. Denn seine Erzählung enthält mehr als eine einfache 
moralpädagogische Volksunterweisung wie sie andere Volksschriftsteller in dieser Zeit veröffentlicht haben. Wie etwa die Jugenderzählung von Gustav Nieritz aus dem Jahre 1849 mit dem Titel Das neue Aschenbrödel oder die Dorferzählung von Joseph Joachim mit dem schlichten Titel Aschenbrödel von 1896, so preist auch Auerbach die bürgerliche Arbeits- und Pflichtenethik, deren vorbildliche Ausübung am Ende mit gesellschaftlicher Anerkennung und sogar mit sozialem Aufstieg belohnt wird. Und auch wenn es in der Erzählung explizit heißt, dass die Dorfbewohner Amrei, d.h. das Barfüßele, für ein „Aschenbuttel“ halten (Auerbach, 1864: 169), erschöpft sich dessen Charakter in diesem vorbildhaften Rollenmodell der tugendhaften und fleißigen Dienstmagd nicht (Wild, 2011: 291f.). Barfüßele wird auch mit den Hauptfiguren aus „Die Gänsemagd“ (KHM 89) oder aus „Die Gänsehirtin am Brunnen“ (KHM 179) assoziiert, mit unschuldigen Königstöchtern, die zur Arbeit als Gänsemagd gezwungen werden, bevor sie nach Jahren der Entbehrung und harten Arbeit sozial wieder rehabilitiert werden. Amrei selbst beschreibt sich als „Salzgräfin“ (Auerbach, 1864: 217) und spielt damit auf das Märchen von der „Prinzessin Mäusehaut“ (KHM 71a) an, in dem die Prinzessin ihren Vater mit Salz vergleicht und deshalb vorübergehend verstoßen wird. Da sie gerne Rätsel aufgibt, erinnert sie die Leser auch unweigerlich an die "Rätselprinzessinnen“ (Feustel, 2004: 282-291), die man aus den Kinder- und Hausmärchen kennt, wie etwa „Das Rätsel“ (KHM 22) oder „Vom klugen Schneiderlein“ (KHM 114). Zudem spricht sie gelegentlich in Märchenzitaten: Sie benutzt die Wendung „Thu' dich auf! Thu' dich auf!“ aus „Simeliberg“ (KHM 142), als sie vor der verschlossenen Tür zum Haus ihrer verstorbenen Eltern steht (Auerbach, 1864: 46), und empfindet ihren ersten Besuch im Gasthaus wie bei „Tischlein deck dich“ (Auerbach, 1864: 230). Auch ihr Gemahl Johannes wird volkspoetisch aufgeladen, wenn er als „Schimmelreiter“ bezeichnet wird (Auerbach, 1864: 189, 222), der im damaligen Volksglauben für Sankt Nikolaus stand oder ganz allgemein Glück verheißen konnte. Da sich Barfüßele auch stets um ihren Bruder Dami kümmert, erinnern die beiden auch an das berühmte Geschwisterpaar Hänsel und Gretel (KHM 15), die sich elternlos durchs Leben schlagen müssen.

Die vielen Märchenanspielungen dienen dazu, die Hauptfigur Amrei zu charakterisieren und ihre Volkstümlichkeit zu illustrieren. Die meisten der wortwörtlichen oder motivischen Übernahmen sowie der verschiedenen Strukturreproduktionen, der Wiederholungen von Handlungsverläufen oder Personenkonstellationen, werden in Auerbachs Erzählung nicht als intertextuelle Referenzen ausgewiesen. Das Verschweigen der Interdependenzen geschieht mit Absicht: Barfüßele soll dem Leser nicht als Märchenfigur vorgeführt werden, sondern als modernes Mädchen aus dem Volk. Unbewusst freilich soll sich der Leser sehr wohl an seine Märchenlektüre erinnert fühlen und ihm dadurch das Gefühl vermittelt werden, Barfüßele schon längst zu kennen.

Gleich mehrfach codiert als Figur der Natur-, resp. Volkspoesie, verkörpert insbesondere Amrei eine positiv konnotierte ursprüngliche, natürliche Anthropologie, die man auch bei vielen Märchenfiguren beobachten kann. Befreit von gesellschaftlichen Konventionen wie etwa Standesschranken, folgt sie - wie auch Johannes - lediglich ihrem reinen und unschuldigen Herzen und legt damit ein vorbildliches Sozial- und Liebesverhalten an den Tag, das auch in der modernen Gesellschaft Bestand haben kann, wie der Ausgang der Erzählung nachdrücklich zeigt: Johannes und Amrei heiraten aus Liebe und setzen sich mit der damit verbundenen Nichtbeachtung ihrer sozialen Stellung über bestehende gesellschaftliche Gepflogenheiten hinweg, wie im Text explizit betont wird. Am Ende 
freilich wird ihre Entscheidung von allen akzeptiert und gelobt, wodurch sich ein märchenhaftes happy end einstellt.

7 Vor allem dieser Erzählverlauf hat bislang den Großteil der Interpreten dazu veranlasst, die Dorfgeschichte Barfüßele als idyllische Sozialutopie zu rezipieren (Hein, 1980: 173-187; Mahlmann-Bauer, 2010: 555-596; Rohde, 2012: 221-243). Die Erzählung lässt sich aber auch als Sozialkritik lesen, wenn man sich vor Augen führt, dass Auerbach die von Amrei verkörperte natürliche Anthropologie auch ins Politische wendet. Amreis ausführlich beschriebene Tagträume (Wild, 2011: 293-300) rekurrieren auf den Naturzustand der Menschheit, der „nichts [...] von den Schranken [hat], die das beengte Leben der Wirklichkeit setzt" (Auerbach, 1864: 52). Die träumerische Vision einer Gesellschaft in Freiheit und Gleichheit wird in der Erzählung jedoch nicht ausführlicher mit den herrschenden gesellschaftlichen Verhältnissen konfrontiert, vielmehr wird ihr Wahrheitsgehalt hervorgehoben und damit neben dem abstrakt-logischen Denken eine alternative Wissensform des Volkes etabliert. Die Frage am Ende des Traums, warum habe „man sich denn das Alles ausgedacht, wenn es nicht wahr" sei (Auerbach, 1864: 53), attestiert Amreis Vision einen Wahrheitswert, der aus der bloßen Existenz der Vision resultiert und auf der Annahme einer natürlichen Anthropologie beruht. Hier artikuliert sich die "Natur" und deshalb ist die Vision wahr und stellt keine subjektiv-willkürliche Phantasterei dar. Die Frage berührt zugleich einen der wichtigsten poetologischen Aspekte der Märchenüberlieferung, den man heute jedoch leicht vergisst, da wir Märchen vorwiegend als Kindererzählungen ansehen. Im 19. Jahrhundert verstand man die Märchen auch als Ausdruck eines alten, lange zurückliegenden volkstümlichen Wissens und Glaubens. So hatten etwa die Brüder Grimm in ihrer Vorrede zu den Kinder- und Hausmärchen Märchen als „alte, verloren geglaubte, in dieser Gestalt aber noch fortdauernde deutsche Mythen" charakterisiert, die „Gedanken über das Göttliche und Geistige im Leben“ aufbewahren und „alte[n] Glaube[n] und Glaubenslehre“ überliefern (Grimm, 2004: XXVII). In diesem Sinne verstand auch Auerbach die gesellschaftliche Funktion der Märchen und attestierte ihnen, dass sie dem deutschen Volk die „tiefste Kunde seines eignen Selbst“ geben würden und in ihnen das „Urthümliche“ dargestellt werde, das „aus der nationalen geheimnißvollen Tiefe“ stamme (Auerbach, 1867: 193). Amreis Träume tradieren also das alte Wissen über die eigene ursprüngliche Gesellschaftsordnung und erinnern an das freiheitliche Wesen des deutschen Volkes, das Standesgrenzen überwinden und ein harmonisches Ganzes bilden kann. Synekdochisch erscheinen Amrei und Johannes aus dieser Perspektive als Symbol für die Lage des deutschen Volkes in der Mitte des 19. Jahrhunderts: $\mathrm{Zu}$ Vorzeiten frei und (politisch) selbstbestimmend, ist es in der Gegenwart geknechtet, wird aber eines kommenden Tages die alten Rechte und Freiheiten wiedererlangen. Unschwer lassen sich hier die liberalen Überzeugungen Auerbachs erkennen.

Bei Eugenie Marlitts erstem Großerfolg, der Goldelse, lässt sich ein ganz ähnliches Verfahren der intertextuellen Codierung beobachten wie bei Auerbach. Auch Marlitt charakterisiert ihre weibliche Hauptfigur als moderne Wiedergängerin tradierter Figuren der Volkspoesie und auch bei ihr lässt sich eine eher versteckt vorgetragene Gesellschaftskritik herausarbeiten (Reiling, 2013: 221-233). Wie in Auerbachs Dorfgeschichte, so wird auch Marlitts Hauptfigur, Elisabeth Ferber, im Roman stets mit ihrem Übernamen "Goldelse“ angesprochen, der ein bestimmtes Merkmal von ihr fokussiert: Während Grimms Rotkäppchen ein rotes Kopftuch trägt, Barfüßele immer barfuß geht und beide davon ihren Namen haben, so hat Goldelse schöne goldene Haare. 
Ihr Name erinnert freilich auch an die Goldmaria aus Bechsteins Märchen „Goldmaria und Pechmaria“. In der Erzählung wird sie auch als Aschenbrödel, als Dornröschen, als Schneewittchen oder auch lediglich als Fee oder Elfe bezeichnet, zudem stellt sie sich als (adlige) Nachkomme einer freiheitsliebenden Zigeunerin heraus und wird als wohltätige, heilige Elisabeth von Thüringen apostrophiert (Marlitt, 1868: 264, 296, 9, 274, 298, 366). Durch diese mehrfache Identifizierung mit Figuren der Volks- bzw. Naturpoesie erhält, wie schon bei Auerbach gesehen, Goldelse ihre Konturen. Anders als Auerbach scheut sich Marlitt jedoch nicht, die Prätexte bzw. die modellgebenden Märchenfiguren explizit zu benennen. Sie tut dies, weil sie sich nicht wie Auerbach auf Märchen bezieht, in denen die Veränderung des sozialen Status der Heldin im Fokus steht, sondern vorwiegend persönliche Merkmale sowie individuelle Tugenden wie Hilfsbereitschaft, Güte oder Schönheit etc. zum Ausdruck bringen will. Der erzählerische Effekt ist in beiden Texten jedoch vergleichbar: Wie schon bei Auerbach gesehen, so geht es auch Marlitt darum, mit und in ihrer Hauptfigur einen bestimmten Menschentypus darzustellen, der sich durch seine natürliche, unverdorbene Lebensart auszeichnet. Das „Naturkind“ aus dem Volk, das in beiden Texten jeweils im Mittelpunkt der Handlung steht, wird durch diese Mehrfachkodierungen zu einer transhistorischen Figur. Die Märchenverweise zeichnen dem intradiegetisch im 19. Jahrhundert lebenden Mädchen eine historische Tiefendimension ein, die es so zeitlos wie aktuell macht. Damit leisten Auerbach und Marlitt Innovatives für die Motivgeschichte des Naturkinds.

Das Naturkind, das man in der Forschung gemeinhin nicht mit diesem zeitgenössischen Namen benennt, sondern mit dem modernen Begriff der „Kindfrau“ oder auch als „Frau aus dem Volk“ bezeichnet, ist ein epochaler Figurentyp, der einem in verschiedenen Ausprägungen in der Literatur des 19. Jahrhunderts begegnet. Während der Begriff bis gegen Ende des 18. Jahrhunderts vor allem den „edlen Wilden“ bezeichnete, der obwohl im Stande der Natur lebend, in moralischer Hinsicht den sittlich verdorbenen Europäern überlegen war, so veränderte sich seit Ende des 18. Jahrhunderts dessen Bedeutung. Seit damals bezeichnet „Naturkind“ vorwiegend ein junges Mädchen, das sich noch in der Pubertät befindet oder sie gerade erst beendet hat, das ein naives, unschuldiges Wesen besitzt und dessen Natürlichkeit sich vielfach darin artikuliert, dass es gerne durch die Natur streift, eng mit Tieren und der Natur verbunden ist und/oder meist über eine musische Begabung verfügt. Das Naturkind hebt sich vom normalen gesellschaftlichbürgerlichen Rahmen ab; Ausdruck kann das etwa in einer besonderen Kleidung finden (oder wie hier in Barfüßigkeit resp. goldenem Haar), oder in Verhaltensweisen, die ansonsten von keinem anderen geteilt werden, wie etwa das auf die Bäume klettern. Das Naturkind kann (muss aber nicht) aus einer naturnahen, also eher einfachen ländlichen Familie stammen, weitaus häufiger stammt es freilich aus einer bürgerlichen Familie, die auf dem Land lebt. Damit wird auch deutlich, dass der Topos des Naturkinds keineswegs in einem naturalistischen Sinne als Abbild einer realen Person zu verstehen ist. Vielmehr ist das Naturkind eine künstlich erdachte Phantasiefigur, eine fingierte Gestalt, die eine natürliche Unschuld und naive Rechtschaffenheit verkörpert, der in der dargestellten bürgerlichen Gesellschaft insofern ein exotischer Status zukommt, als sie dort eine singuläre Erscheinung ist.

Goethes Mignon, aus Wilhelm Meisters Lehrjahren (1795-1796), dürfte das wohl bekannteste Beispiel für ein Naturkind sein, dem Effi Briest, aus dem gleichnamigen Roman Theodor Fontanes (1894-1896) nur wenig nachsteht. In August von Kotzebues Komödie Die Indianer in England (1790) verkörpert die indische Fürstentochter Gurli die exotische Variante des 
Naturkindes, die somit für die angesprochene geschlechtliche Verschiebung auch innerhalb des Diskurses des „edlen Wilden“ stehen kann. Heinrich Claurens erfolgreiche Erzählung Mimili von 1818 gibt mit den religiösen Konnotationen der Hauptfigur einen weiteren wichtigen imagologischen Traditionszusammenhang $\mathrm{zu}$ erkennen, der sich literarisch als Bukolik und Idyllik manifestiert. Auch bei Karl Gutzkow (Die Frau aus dem Volk oder Imagina Unruh), Adalbert Stifter (etwa Die Narrenburg), Gottfried Keller (Berlocken ) oder Josef Rank (Else, das Ducatenkind) taucht das Naturkind auf, und auch Johanna Spyri rekurriert mit ihrem Roman von 1880, Heidis Lehr- und Wanderjahre, auf diesen Topos, der romantische Kindheitsvorstellungen weiterführt, wie eine Erzählung Auerbachs bezeugt. In der Dorfgeschichte Die Frau Professorin wird die Bauern- und Wirtstochter Lorle, die durch Heirat zur Frau Professorin wird, im Duktus der Romantik ebenfalls als Naturkind beschrieben. Lorle verkörpere, so heißt es, den „Typus des Urmenschlichen, des ursprünglich Vollkommenen, an sich Vollendeten“, der „unberührt von den Zwiespältigkeiten der Geschichte und der Bildung" geblieben sei (Auerbach, 1864: 241). Hier zeigt sich mit aller Deutlichkeit, dass das Naturkind im 19. Jahrhundert als Ursprungschiffre diente, der man sentimentalisch nachhing. Als Verkörperung der ursprünglichen Ganzheit oder integren Totalität des Menschen repräsentiert das Naturkind eine Entwicklungsstufe, die dem Bildungsbürger des 19. Jahrhunderts abhanden gekommen war, zu der er sich aber zurücksehnte. Und so fungierte das Naturkind in der Literatur des 19. Jahrhunderts als Reflexionsfigur über den Zivilisationsprozess und mithin über das durch die vielfältigen Formen der sozialen und gesellschaftlichen Differenzierungen prekäre Verhältnis von Natur und Kultur.

11 Literarisch wird dieses Verhältnis ganz unterschiedlich gestaltet, wobei sich zwei grundlegende Varianten ausmachen lassen: Wie etwa im Falle von Mignon oder Imagina Unruh, der Hauptfigur aus Gutzkows gleichnamiger Erzählung, kann das Naturkind als Verkörperung des Fremden oder des kulturell Anderen auftreten, das aufgrund seiner Fremd- oder Wildheit domestiziert werden muss. Die Integration misslingt jedoch und das Naturkind, das in diesem Falle somit eher als Bedrohung der Gesellschaft zu deuten ist, stirbt. Es gibt aber auch den weitaus häufiger anzutreffenden Fall des domestizierten Naturkinds, das sich in die Gesellschaft einzugliedern vermag; eines der berühmtesten Beispiele hierfür ist wohl Emmy von Rhodens Trotzkopf von 1886. Auch Barfüßele und Goldelse gehören $\mathrm{zu}$ dieser Kategorie, wobei sie wiederum eine spezielle Variante verkörpern. Zugespitzt könnte man es so formulieren: Die Integration dieser beiden Naturkinder gelingt, weil sich die Gesellschaft verändert und sich ihnen anpasst. Beiden Figuren wohnt das Potential der Erneuerung oder Revitalisierung inne, das man im 19. Jahrhundert der Volkspoesie insgesamt attestiert hatte. Die einfachen Mädchen symbolisieren den in die Gegenwart hereingeholten Ursprung, der eine Korrektur der gesellschaftlichen Verirrungen bewirken kann. Wo Auerbach den Blick auf die gesellschaftlich-sozialen Zustände lenkt und mit der Heirat von Barfüßele symbolisch die neue, aber im Grunde ja alte und ursprüngliche Gesellschaftsordnung zum Ausdruck bringt, die am Ende wieder hergestellt wird, so lenkt Marlitt den Blick auf die Geschlechter: die Einordnung von Goldelse in die Gesellschaft gelingt nur, weil sich auch ihr zukünftiger Gemahl verändert. Rudolf von Walde muss seine rationale und ökonomische Effizienz erst in eine humane, sympathetische Gewandung hüllen, damit er und Goldelse sich näherkommen können. Wie in allen Romanen von Marlitt, muss auch in der Goldelse der Mann, der zunächst auf die Heldin abstoßend und unsympathisch wirkt, gefühlvoller und damit sozial umgänglich werden, um auch von der Frau begehrt, geliebt und geschätzt $\mathrm{zu}$ werden. Marlitts Romane führen also in immer wieder neuen 
Variationen vor, wie die moderne Kultur, die durch die Männer symbolisiert wird, mit der von den Mädchen verkörperten Natur zusammenfinden kann, wodurch sich eine stabile Gemeinschaft bildet. Das funktioniert nur, wenn die Männer teilweise umdenken und von den Mädchen lernen - oder anders formuliert: der moderne, zivilisierte Mensch muss sich vom natürlichen Menschen, der sich in den Kindfrauen des 19. Jahrhunderts und auch in den Märchenfiguren verbirgt, Aspekte bewahren und diese präsent halten.

Goldelse und Barfüßele erweisen sich damit als „Heil- oder Korrekturmittel“ von gesellschaftlichen Fehlentwicklungen. Diese korrektive Funktion erhalten die Naturkinder vor allem durch ihre volkspoetische Aufladung. Während die Intertextualität zum einen als Beglaubigung für die Wahrhaftigkeit der dargestellten natürlichen Anthropologie dient, hält sie zum anderen aber auch stets die Differenz zwischen (literarisch dargestelltem) Ideal und (außerliterarischem) gesellschaftlichen Ist-Zustand aufrecht. Den Märchenzitaten kommt in beiden Erzählungen konstitutive Bedeutung zu, da erst sie eine kultur- und zeitkritische Lesart eröffnen. Freilich erschließt sich eine solche Lesart nur, wenn man Märchen nicht als bürgerlich-moralistische Literatur versteht, mit denen Kindern das rechte Verhalten eingeimpft werden soll. Erst wenn man Märchen als nationale Volkspoesie wahrnimmt und sie somit als historische, anthropologische Quellentexte früherer Epochen rezipiert, können sie als kritische Hintergrundfolie der eigenen Gegenwart dienen.

Die Bedeutung der intertextuellen Referenzen wird also durch poetologische Ansichten determiniert, die dem literarischen Text vorausliegen. Ich möchte deshalb abschließend den Blick kurz auf diesen theoretischen Diskurs richten, um das bisher dargestellte noch einmal aus einer anderen Perspektive zu beleuchten und damit zugleich auf einen vergessenen Aspekt in der Poetikgeschichte des 19. Jahrhunderts aufmerksam zu machen. Für die Konstitutionsphase des Realismus, in der die Texte Auerbachs und Marlitts erscheinen, spielen theoretische Überlegungen zum Wesen der Volkspoesie eine bedeutende Rolle, die man bis heute jedoch kaum näher erforscht hat ${ }^{1}$. Ich beschränke mich in meinen Ausführungen lediglich auf Auerbachs 1846 erschienene Poetik Schrift und Volk, die paradigmatisch die vielfältigen literarhistorischen Konstellationen jener Zeit zum Ausdruck bringt.

14 In seiner Poetik entwirft Auerbach u. a. das Modell eines zeitgenössischen Autors, den er als Volksschriftsteller charakterisiert und dementsprechend in die Tradition der Volkspoesie einordnet. Das von Auerbach entworfene Autormodell orientiert sich an Positionen, die Jacob Grimm und Achim von Arnim zu Beginn des 19. Jahrhunderts in ihrem Streit über die Natur- und Kunstpoesie vertreten hatten. In den 1808 in Achim von Arnims Zeitung für Einsiedler erschienenen Gedanken: wie sich die Sagen zur Poesie und Geschichte verhalten hatte Jacob Grimm die Volks- und Kunstpoesie in produktionsästhetischer Perspektive unterschieden. Die Volkspoesie beschrieb er als „unwillkührlich[e]“, gleichsam mirakulös entstehende Dichtung, die aus dem Volk heraus entstehe, ohne dass sich eine konkrete Einzelperson als Urheber identifizieren ließe, die Kunstpoesie hingegen entspringe durch einen bewussten Schöpfungsakt eines einzelnen Individuums (Grimm, 1808: 151-156). Beide Poesien galten ihm, wie auch schon Herder, als historisch aufeinander abfolgende Phasen, wobei er die Naturpoesie (der Vergangenheit) als „Poesie der Ungebildeten“, die (moderne) Kunstpoesie hingegen als „Poesie der Gebildeten“ charakterisiert und diesen Verlauf als degenerierte Literaturgeschichte deutete. Von Arnim bestritt diese kulturgeschichtliche Deutung und verstand beide Poesien vielmehr als Beschreibungen des kreativen Prozesses und glaubte, 
dass diese Arten des Dichtens somit auch immer wieder in verschiedenen Epochen und damit gleichzeitig auftreten können (Steig, 1904: 108-144). Auerbach führt beide Ansichten zusammen und amalgamiert die kategorial verschieden aufgefassten Konzepte der Natur- und Kunstpoesie im Zwittermodell einer „natürlichen Kunstpoesie“, die er dem zeitgenössischen Literaturbetrieb als Idealform anpreist. In seinem Literaturverständnis kommt dem schöpferischen Individuum bzw. der dichterischen Individualität nur marginale Bedeutung $\mathrm{zu}$, der Dichter ist kein autonomes, kreatives Originalgenie, sondern ein „Prophet [...]“, der das „innerste Leben der Wirklichkeit“ schaut und das „Ewige [...] in seiner endlichen Erscheinung“ verkündet: (Auerbach, 1864: 15) „Das Volksthümliche verlangt ein völliges Zurücktreten des Autors und es ist nicht ohne Bedeutung, daß wir oft von den besten Gebilden in diesen Kreisen die Namen der Urheber nicht mehr kennen." (Auerbach, 1846: 51) Der moderne Volksschriftsteller soll also die Nachfolge des früheren, anonymen Erzählerkollektivs „Volk“ antreten.

Während Auerbach auf der einen Seite von einer individuellen Dichterverehrung Abstand nimmt, hängt er ihr auf der anderen Seite dennoch an. Denn die Rede vom poeta vates verweist auch zugleich auf die gesellschaftlich elitäre Stellung des Dichters. Entsprechend sieht Auerbach diesen durchaus als außergewöhnliche und besondere Persönlichkeit an und definiert ihn als "welthistorisches Individuum “ im Sinne von Hegels Geschichtsphilosophie, das sich deutlich von der breiten Masse abhebt, indem es seine eigenen Ziele verfolgt, aber gleichzeitig dadurch den Weltgeist realisiert: „Nur die größten Geister vermögen es, sich so in sich zu vertiefen, daß sie in sich selber den Gesammtgehalt ihrer Epoche fassen und harmonisch gestaltet heraustreten lassen." (Auerbach, 1846: 14) Der Dichter muss nach Auerbach das Volk mit all seinen Facetten und Schattierungen kennen, aber gleichzeitig auch eine gewisse Distanz zu ihm haben, um die im Wesen des Volkes gründenden gesellschaftlichen Entwicklungspotentiale aufzeigen und den Weg zu einer besseren Gesellschaft weisen zu können. Während frühere Dichter Märchen und weitere volkspoetische Erzählformen verwendet haben, brauchen die modernen volkstümlichen Dichter neue Formen, da die Menschheit ihre ursprüngliche Naivität verloren und höhere intellektuelle Fähigkeiten entwickelt habe. Die Zeit, in der die Menschen ihre Glaubens- und Naturlehren in Märchen und Mythen eingekleidet haben, sei, wie Auerbach mit einem Seitenhieb gegen romantische und vormärzliche Kunstmärchen konstatiert, dementsprechend vorbei. Der neue „freie Geist läßt sich nicht mehr in die Behausung der Märchen zurücklocken“ (Auerbach, 1846: 63), sondern brauche neue Formen, wie sie etwa seine eigenen Dorfgeschichten oder Marlitts Romane darstellen.

Auerbachs Barfüßele und etwas weniger auch Marlitts Goldelse künden von diesem Bemühen, im Anschluss an die Volkspoesie und als deren Fortführung eine moderne Literatur zu schaffen, die - durchaus epochentypisch - im Dienst einer liberalen Nationalpädagogik steht, die auf die Verwirklichung eines freiheitlichen und vereinten deutschen Rechtsstaates hinarbeitet. Beide Autoren setzen dabei auf die Kraft der Evolution und nicht der Revolution und verstehen ihre ästhetische Erziehung als Erneuerung aus dem Geist der Nation: deshalb zitieren sie die überlieferten Volksmärchen. Möglicherweise waren ihre Erzählungen auch gerade deswegen solche Bucherfolge. 


\section{BIBLIOGRAPHIE}

AUERBACH Berthold (1846), Schrift und Volk.

AUERBACH Berthold (1864), Berthold Auerbach's gesammelte Schriften, Zweite Gesammtausgabe, Stuttgart: Cotta.

AUERBACH Berthold (1867), „Jakob Grimm“, B. Auerbach, Deutsche Abende (185-202), N. F., Stuttgart: Cotta.

AUERBACH Berthold, „Jakob Grimm“, B. Auerbach, Erzählungen. Eine Auswahl (103-116) Mit einem Nachruf von Otto Brahm und einer Bleistiftzeichnung von Carl Spitzweg, ausgewählt u. mit einem Nachwort versehen v. Walter Hagen, Marbach a. N., 1962.

BETTELHEIM Anton (1907), Berthold Auerbach. Der Mann. Sein Werk - Sein Nachlaß, Stuttgart: Cotta.

BONTER Urszula (2005), Der Populärroman in der Nachfolge von E. Marlitt. Wilhelmine Heimburg, Valeska Gräfin Bethusy-Huc, Eufemia von Adlersfeld-Ballestrem, Würzburg: Königshausen und Neumann. FEUSTEL Elke (2004), Rätselprinzessinnen und schlafende Schönheiten. Typologie und Funktionen der weiblichen Figuren in den Kinder- und Hausmärchen der Brüder Grimm, Hildesheim: Olms.

GRIMM Jacob und Wilhelm, Werke, Abt. III : Gemeinsame Werke, Bd. 43 : Kinder- und Haus-Märchen 1819/1822, Bd. 1. Neu hg. v. Hans-Jörg Uther, Hildesheim, Zürich, New York: Olms, 2004 [Nachdruck].

GRIMM Jakob, „Gedanken: wie sich die Sagen zur Poesie und Geschichte verhalten“, Zeitung für Einsiedler (151-156), $\mathrm{n}^{\circ}$ 19/20, 4. und 7. Juni 1808.

HEIN Jürgen (1980), „Berthold Auerbach: Barfüßele (1856). Dorfgeschichte als Rettung der ,Schönheit des Heimlichen und Beschränkten“" (173-187), Horst Denkler (dir.), Romane und Erzählungen des Bürgerlichen Realismus. Neue Interpretationen, Stuttgart: Reclam.

MAHLMANN-BAUER Barbara (2010), „Sozial- und Erzählstruktur in Werken Jeremias Gotthelfs und Berthold Auerbachs“, Achim Aurnhammer, Wilhelm Kühlmann \& Hansgeorg Schmidt-Bergmann (dir.), Von der Spätaufklärung zur Badischen Revolution. Literarisches Leben in Baden zwischen 1800 und 1850 (555-596), Freiburg: Rombach.

MARLITT Eugenie (1868), Goldelse, Leipzig: Ernst Keil.

REGENBOGEN Albrecht (2006), Barfüßele auf seinem Weg in die Welt. Berthold Auerbachs erfolgreichste Dorfgeschichte wird 150, Eine Dokumentation, Horb a. N.

REILING Jesko (2013), „,Wird sie, das Aschenbrödel von der Kaiseralm, jemals einen Brautkranz tragen?' Das Aschenputtel-Märchen in Liebesromanen seit dem 19. Jahrhundert“, Eva Parra Membrives, Albrecht Classen (dir.), Literatur am Rand / Literature on the Margin. Perspektiven der Trivialliteratur vom Mittelalter bis zum 21. Jahrhundert / Perspectives of Trivial Literature from the Middle Ages to the 21st Century (221-233), Tübingen: Narr.

REILING Jesko (2012), „Eine Literatur für alle. Auerbach und die Volkspoesie“, J. Reiling (éd.), Berthold Auerbach (1812-1882). Werk und Wirkung (97-120), Heidelberg: Winter. 
ROHDE Carsten (2012), „Zwischen Sentimentalität und Utopie. Zur semantischen und ästhetischen Codierung von Liebe bei Berthold Auerbach“, J. Reiling (dir.), Berthold Auerbach (1812-1882). Werk und Wirkung (221-243), Heidelberg: Winter.

SCHEUFFELEN Thomas (1986), Berthold Auerbach 1812-1882, Marbach a. N.

STEIG Reinhold (1904), Achim von Arnim und die ihm nahe standen, Bd. 3: Achim von Arnim und Jacob und Wilhelm Grimm, Stuttgart, Berlin: Cotta.

WILD Bettina (2011), Topologie des ländlichen Raums. Berthold Auerbachs „Schwarzwälder

Dorfgeschichten" und ihre Bedeutung für die Literatur des Realismus, Würzburg: Königshausen und Neumann.

\section{NOTES}

1. Zurzeit arbeite ich an einer größeren Studie zu diesem Themenkomplex, die bestrebt ist, diese Lücke auszufüllen.

\section{RÉSUMÉS}

Berthold Auerbachs Barfüßele (1856) und Eugenie Marlitts Goldelse (1866) gehören zu den erfolgreichsten Romanen des 19. Jahrhunderts. Explizit erklärten sich die Zeitgenossen den Erfolg dieser Werke mit der „Volksthümlichkeit“ (Rudolf Gottschall) der darin behandelten Stoffe, wobei vor allem der ans „Aschenputtel“ erinnernde Handlungsverlauf (beider Romane) dieses Urteil hervorgerufen haben dürfte. Beide Romane zitieren darüber hinaus eine Vielzahl weiterer Märchen und Sagenerzählungen und entwerfen damit ein erklärungsbedürftiges intertextuelles Geflecht.

Der vorliegende Beitrag möchte aufzeigen, dass und wie sich beide Erzählungen nicht nur auf der (motivischen, stilistischen, etc.) Textoberfläche, sondern auch in poetologischer Hinsicht in die Tradition der Volkspoesie einreihen. Beide Werke entwerfen durch die vielen Märchenbezüge einerseits „modern verkleidete Archetypen“ (Hermann Bausinger), andererseits eröffnen die anzitierten Prätexte für den jeweiligen Posttext einen erweiterten Deutungshorizont, der durch die von Johann Gottfried Herder angestoßene, also rund 80 Jahre zurückreichende Diskussion über das Verhältnis von Natur- und Kunstpoesie abgesteckt ist. Beide Texte lassen sich im Herder'schen Sinne als Versuche verstehen, eine moderne Form der Volkspoesie, eine zeitgemäße Variante der Volksmärchen, zu finden. Als volkstümliche Literatur wollte Auerbach seine Schwarzwälder Dorfgeschichte(n) verstanden wissen, die wie Grimms Kinder- und Hausmärchen als „ächte Poesie“ (Vorrede, Erstauflage der KHM) das wahre Leben darstellen und das deutsche „Geistes- und Gemüthsleben“ (Auerbach) zum Ausdruck bringen und befördern. Auerbachs und Marlitts Werke schreiben sich in einen heute von der Forschung kaum mehr beachteten Diskurs ein, der aber - wie der Erfolg beider Werke belegt - bis in die 1860er Jahre intensiv geführt wurde.

Barfüßele (1856) de Berthold Auerbach et Goldelse d'Eugenie Marlitt (1866) figurent parmi les romans allemands les plus célèbres $d u$ xIx siècle. Leurs contemporains expliquaient 
ouvertement le succès de ces œuvres par le « caractère populaire » (Rudolf Gottschall) des sujets qu'elles traitaient, ce jugement étant probablement dû aux similitudes que présente l'action des deux romans avec le conte de Cendrillon. Tous deux citent par ailleurs un grand nombre de contes et de légendes, créant ainsi un entrelacement intertextuel qui demande à être explicité.

La présente communication se propose de montrer comment ces deux récits s'inscrivent dans la tradition de la poésie populaire (Volkspoesie), non seulement à la surface du texte lui-même (motifs et procédés stylistiques, etc.), mais aussi d'un point de vue poétologique. Par les multiples références aux contes, ces deux œuvres donnent naissance, d'une part, à des «archétypes en habits d'aujourd'hui » («modern verkleidete Archetypen », Hermann Bausinger); d'autre part, les pré-textes cités ouvrent, pour chacun de leurs post-textes, un horizon de lecture élargi et jalonné par la discussion initiée exactement 80 ans plus tôt par Johann Gottfried Herder et portant sur les rapports entre poésie de nature (Naturpoesie) et poésie d'art (Kunstpoesie).

Ces deux textes peuvent être considérés comme des tentatives, au sens où l'entendait Herder, pour trouver une forme moderne de la poésie populaire, une variante des contes populaires qui serait en adéquation avec son époque. Auerbach voulait qu'on voie de la littérature populaire dans ses «Schwarzwälder Dorfgeschichten " (Récits villageois de la forêt noire) qui, au même titre que les Contes de l'enfance et du foyer des frères Grimm, représentent la vie véritable comme « de la

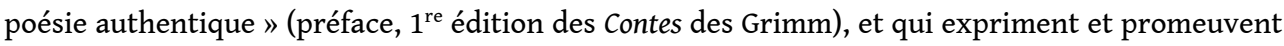
«l'esprit et l'âme » allemands (Auerbach).

Les œuvres respectives d'Auerbach et de Marlitt s'inscrivent dans un discours auquel les travaux de recherche actuels n'accordent plus guère d'importance, mais qui eut toute sa place jusque dans les années 1860, ce qu'atteste le succès de ces romans.

Barfüßele (1856) by Berthold Auerbach and Goldelse (1866) by Eugenie Marlitt are among the most famous novels of the $19^{\text {th }}$ century. Contemporaries ascribe the success of these books to the " Volksthümlichkeit" (Rudolf Gottschall) covered in these oeuvres. Similarities, in particular to the storyline of "Cinderella", might have caused this impression. Both novels additionally quote various other fairy tales and legends/myths, creating thereby an inter-textual meshwork.

In the following presentation I am going to argue that both narratives are akin to folk poetry (Volkspoesie) not only in relation to the text format (motifs and style etc.) but also with regards to its poetics, and I want to demonstrate how this is achieved in both narratives. On one hand, both novels portray "modern verkleidete Archetypen" [archetypes in modern disguise] (Hermann Bausinger), and on the other the quoted text passages do provide ample room for interpretation owing to the embedded fairy tale references framed by the discussion about the relation of nature and art poetry [Natur- und Kunstpoesie] (initiated 80 years before by Johann Gottfried Herder). Both narratives can be thought of as attempts to find a modern form of folk poetry or a contemporary variant of popular fairy tales. Auerbach wanted his Schwarzwaelder Dorfgeschichte (n) [Schwartzwald Village Tales] to be recognized as popular and genuine literature just like Grimm's Kinder- und Hausmärchen that describes "real" German life and mindset ("ächte Poesie"). The stories were intended to educate the entire German nation. The works of Auerbach and Marlitt are part of a discourse that seems to go largely unnoticed by today's research, but was nevertheless intensely debated until the 1860 s. 


\section{INDEX}

Keywords : folk poetry, natural poetry, art poetry, Berthold Auerbach, Eugenie Marlitt, fairy tale, Schwarzwald Village Tales, 19th century, realism, child of Nature, intertextuality Mots-clés : poésie populaire, poésie de nature, poésie d'art, Berthold Auerbach, Eugenie Marlitt, conte, récits villageois de Forêt-Noire, xixe siècle, réalisme, enfant de la nature, intertextualité Schlüsselwörter : Volkspoesie, Naturpoesie, Kunstpoesie, Berthold Auerbach, Eugenie Marlitt, Märchen, Schwarzwälder Dorfgeschichten, 19. Jahrhundert, Realismus, Naturkind, Intertextualität

\section{AUTEUR}

\section{JESKO REILING}

Université de Berne 